HUPD-9613

KEK-TH-489

August 1996

revised version February 1997

\title{
Test of the chiral structure and FCNC in the quark sector by radiative $B$ meson decays
}

\author{
L. T. Handoko $\mathrm{t}$ \\ Department of Physics, Hiroshima University \\ 1-3-1 Kagamiyama, Higashi Hiroshima - 739, Japan \\ T. Yoshikawa 8 \\ National Laboratory for High Energy Physics (KEK) \\ Tsukuba - 305, Japan
}

\begin{abstract}
We study the effects of a vector-like SU(2) quark doublet as a fourth generation. In this model we examine the chiral structure and the FCNC in the quark sector by using radiative $B$ meson decays in the allowed region for parameters from $R_{b}=\Gamma_{q q} / \Gamma_{\text {had. }}$. We compute the ratio $R=\operatorname{Br}(b \rightarrow d \gamma) / \operatorname{Br}(b \rightarrow s \gamma)$ in the model which realizes a different chiral structure as well as FCNC. The constraints has been extracted from the experimental results of $B$ meson decays, the $T_{\text {new }}$ parameter of oblique corrections and $R_{b}$. Under the natural assumption that the violation of the $V-A$ structure in the light-quark sector is small, we can determine the allowed region for most of the mixings parameters and the vector-like quark masses. We show that there will be significant deviations in $R$ from the SM prediction due to the FCNC's and the violation of the $V-A$ structure.
\end{abstract}

\footnotetext{
*On leave from P3FT-LIPI, Indonesia. E-mail address : handoko@theo.phys.sci.hiroshimau.ac.jp

${ }^{\dagger}$ E-mail address : yosikawa@theory.kek.jp
} 


\section{INTRODUCTION}

The unitarity of the Cabibbo-Kobayashi-Maskawa (CKM) matrix and the universal $V-A$ structure of the interactions between the $S U(2)_{L}$ gauge bosons, quarks and leptons are two of the remarkable predictions of the $S U(2)_{L} \times U(1)_{Y}$ electroweak interactions. Deviations from these predictions would indicate new physics which should be directly detected in future high-energy experiments. At present, radiative $B$ decays provide an important test of the Standard Model (SM) and provide a sensitive probe of physics beyond the SM. The current result of the CLEO collaboration [[] gives a measurement of $\operatorname{Br}(b \rightarrow s \gamma)$, and in the future it is expected that the $\operatorname{Br}(b \rightarrow d \gamma)$ will also be determined. There is also an effort to search for flavor changing neutral currents (FCNC) in $B$ meson decays [2]. On the other hand, there were also discrepancies between the SM prediction for and experimental measurement of $R_{b}$, which is also a probe of new physics [3] 6 . These experiments provide further motivation of this study. To explain the discrepancy many extensions of the SM have been presented. As the simplest extension of the SM, models that explain the data by using a new flavor mixing from a vector-like quark as a fourth generation have been proposed by Ref. [6, 7]. Here, we introduce an $S U(2)$ doublet of vector-like quarks in addition to the ordinary quarks in the SM. In the previous work of Ref. [7] one of the authors showed that there is an allowed region for new flavor mixing between right handed third generation and the fourth generation which is consistent with satisfies the measured value of $R_{b}$. In that case the mixing parameters must be large. If $R_{b}$ is consistent with the SM prediction, their region for large mixing remains.(See Fig. 1.) We interested in the new allowed region. This provides a real model for the deviation of $V-A$ chiral structure in the charged-current $(\mathrm{CC})$ sector as pointed out in Ref. [8]. At the same time, this also provides FCNC's in up and down quark sectors at once [9, 10. In this work, we examine whether the region remains after including the constraints from radiative

\footnotetext{
${ }^{1}$ The latest report [4 point that the experimental result is consistent with the SM prediction. However the 1996 world average value of $R_{b}$ [5] is not still consistent.
} 
$B$ decays and oblique corrections.

Introducing a doublet vector-like quarks will violate the $V-A$ structure of the SM. This possible violation could provide useful information on new properties of the quarks at higher energy scales as well as the origin of parity violation [8]. Anyway, the induced FCNC due to the addition of the singlet or doublet vector-like quark has been studied by many authors, for example in [9, 11]. In this paper, we study these FCNC's by using the ratio $R=\operatorname{Br}(b \rightarrow d \gamma) / \operatorname{Br}(b \rightarrow s \gamma)$ which is a sensitive probe to study the violation of CKM matrix as pointed out in [12]. The constraints for the model are extracted from the experimental measurements of $b \rightarrow s \gamma$ process, the $T_{\text {new }}$ parameter of oblique corrections and also $R_{q}=\Gamma_{q q} / \Gamma_{\text {had. }}$. Further constraints will be obtained under the natural assumption that the violation of $V-A$ structure in the light-quarks sector is small.

This paper is organized as follows. In Sec II we briefly describe the model and show how the violation of the $V-A$ structure and FCNC's appear. In Sec.II], we review the constraints from $R_{b}$ on the mixing parameters. In Sec.IV, we describe the radiative $B$ decays in the model. In Sec. $\square$, some constraints from the experimental results for the vector-like quark masses, the size of FCNC and the CKM matrix for right-handed quarks are given. Before going to the conclusions, in Sec.VI the numerical results which show the effects on $R$ are presented.

\section{THE MODEL}

We study the SM extended with an $S U(2)$ vector-like quark doublet as a fourth generation. In this extension, the charge assignments of the quark sector under the electroweak symmetry $S U(2)_{L} \otimes U(1)_{Y}$ are,

$$
\begin{aligned}
& \tilde{Q}_{L}^{i}=\left(\begin{array}{c}
\tilde{u}^{i} \\
\tilde{d}^{i}
\end{array}\right)_{L} \rightarrow\left(2, \frac{1}{3}\right), \\
& \tilde{u}_{R}^{i} \quad \rightarrow\left(1, \frac{4}{3}\right) \text {, } \\
& \tilde{d}_{R}^{i} \quad \rightarrow\left(1,-\frac{2}{3}\right), \\
& \tilde{Q}_{L / R}^{4}=\left(\begin{array}{c}
\tilde{u}^{4} \\
\tilde{d}^{4}
\end{array}\right)_{L / R} \rightarrow\left(2, \frac{1}{3}\right) \text {. }
\end{aligned}
$$


Because the vector-like quarks are an $S U(2)$ doublet, the left-handed and righthanded chiralities have the same charge under an $S U(2)_{L}$ transformation. The tilde on the fields denotes weak-eigenstate quark fields, $i$ denotes the flavor, and, as usual the chiralities are $Q_{L / R} \equiv\left(1 \mp \gamma_{5}\right) Q / 2$.

There is an ambiguity in reproducing the masses of the vector-like quarks, but the study should not be altered with the choice. In this paper we adopt the same procedure and notations as Ref.[11]. We consider one Higgs doublet which is the same as in the SM and introduce explicit bare-mass terms for the vector-like quarks. Then, the Lagrangian for the Yukawa sector becomes,

$$
\mathcal{L}_{Y}=-f_{d}^{\alpha i} \tilde{Q}_{L}^{\alpha} \phi \tilde{d}_{R}^{i}-f_{u}^{\alpha i} \tilde{Q}_{L}^{\alpha} \tilde{\phi} \tilde{u}_{R}^{i}-f^{\alpha 4} v^{\prime} \tilde{Q}_{L}^{\alpha} \tilde{Q}_{R}^{4}+\text { h.c. }
$$

with $i=1,2,3$ and $\alpha=1,2,3,4$. In order to obtain the masses and mass eigenstates, we must diagonalize the Yukawa couplings above as follows:

$$
\begin{aligned}
& D_{L}\left(f_{d}^{\alpha i \frac{v}{\sqrt{2}},} f^{\alpha 4} v^{\prime}\right) D_{R}^{\dagger}=m_{d}, \\
& U_{L}\left(f_{u}^{\alpha i \frac{v}{\sqrt{2}},}, f^{\alpha 4} v^{\prime}\right) U_{R}^{\dagger}=m_{u},
\end{aligned}
$$

where $m_{d}$ and $m_{u}$ are the diagonalized mass matrices and $D_{L / R}$ and $U_{L / R}$ are $4 \times 4$ unitary matrices which relate the weak-eigenstates to the mass-eigenstates as,

$$
\begin{aligned}
& \tilde{d}_{L / R}^{\alpha} \equiv D_{L / R}{ }^{\alpha \beta} d_{L / R}{ }^{\beta}, \\
& \tilde{u}_{L / R}^{\alpha} \equiv U_{L / R}{ }^{\alpha \beta} u_{L / R}{ }^{\beta},
\end{aligned}
$$

where $\alpha$ and $\beta$ denote the flavor for four generations. Note that $f^{\alpha 4}$ in both the up and the down sectors must have same value in the third term of Eq. (1), because the term show the $S U(2)$ invariant mass of vector-like doublet quark. Hence there is a relation between the up and the down sector as follows:

$$
f^{\alpha 4} v^{\prime} \equiv\left(D_{L}^{\dagger}\right)^{\alpha \beta} m_{d}^{\beta} D_{R}^{\beta 4}=\left(U_{L}^{\dagger}\right)^{\alpha \beta} m_{u}{ }^{\beta} U_{R}^{\beta 4} .
$$

Here, we give a brief description of how the violation of the $V-A$ structure is realized. As an example, we show only the $W^{ \pm}$and $Z$ sectors. The original 
interactions in the weak-eigenstates are,

$$
\begin{aligned}
& \mathcal{L}_{Z}=\frac{g}{2 \cos \theta_{W}}\left[\overline{\tilde{u}}_{L}^{\alpha} \gamma^{\mu}\left(1-\frac{4}{3} \sin ^{2} \theta_{W}\right) \tilde{u}_{L}^{\alpha}+\overline{\tilde{u}}_{R}^{i} \gamma^{\mu}\left(-\frac{4}{3} \sin ^{2} \theta_{W}\right) \tilde{u}_{R}^{i}\right. \\
& +\overline{\tilde{u}}_{R}^{4} \gamma^{\mu}\left(1-\frac{4}{3} \sin ^{2} \theta_{W}\right) \tilde{u}_{R}^{4} \\
& +\overline{\tilde{d}}_{L}^{\alpha} \gamma^{\mu}\left(-1+\frac{2}{3} \sin ^{2} \theta_{W}\right) \tilde{d}_{L}^{\alpha}+\overline{\tilde{d}}_{R}^{i} \gamma^{\mu}\left(\frac{2}{3} \sin ^{2} \theta_{W}\right) \tilde{d}_{R}^{i} \\
& \left.+\overline{\tilde{d}}_{R}^{4} \gamma^{\mu}\left(-1+\frac{4}{3} \sin ^{2} \theta_{W}\right) \tilde{d}_{R}^{4}\right] Z_{\mu}, \\
& \mathcal{L}_{W^{ \pm}}=\frac{g}{\sqrt{2}}\left(\overline{\tilde{u}}_{L}^{\alpha} \gamma^{\mu} \tilde{d}_{L}^{\alpha}+\overline{\tilde{u}}_{R}^{4} \gamma^{\mu} \tilde{d}_{R}^{4}\right) W_{\mu}^{ \pm}+\text {h.c. },
\end{aligned}
$$

because the left and right handed fourth generation quarks are in an $S U(2)$ doublet. The transformation into the mass-eigenstates gives,

$$
\begin{aligned}
& \mathcal{L}_{Z}=\frac{g}{2 \cos \theta_{W}}\left[\bar{u}_{L}^{\alpha} \gamma^{\mu}\left(1-\frac{4}{3} \sin ^{2} \theta_{W}\right) u_{L}^{\alpha}+\bar{u}_{R}^{\alpha}\left(\gamma^{\mu}-\frac{4}{3} \sin ^{2} \theta_{W}\right) u_{R}^{\alpha}\right. \\
& +\bar{u}_{R}^{\alpha} \gamma^{\mu}\left(U_{R}^{\alpha 4} U_{R}^{\dagger}{ }^{4 \beta}\right) u_{R}^{\beta} \\
& +\bar{d}_{L}^{\alpha} \gamma^{\mu}\left(-1+\frac{2}{3} \sin ^{2} \theta_{W}\right) d_{L}^{\alpha}+\bar{d}_{R}^{\alpha} \gamma^{\mu}\left(\frac{2}{3} \sin ^{2} \theta_{W}\right) d_{R}^{\alpha} \\
& \left.+\bar{d}_{R}^{\alpha} \gamma^{\mu}\left(-D_{R}^{\alpha 4} D_{R}^{\dagger}{ }^{4 \beta}\right) d_{R}^{\beta}\right] Z_{\mu} \\
& \mathcal{L}_{W^{ \pm}}=\frac{g}{\sqrt{2}}\left[{\overline{u_{L}}}^{\alpha} \gamma^{\mu}\left(U_{L}^{\alpha \delta} D_{L}^{\dagger}{ }^{\delta \beta}\right) d_{L}^{\beta}+{\overline{u_{R}}}^{\alpha} \gamma^{\mu}\left(U_{R}^{\alpha 4} D_{R}^{\dagger}{ }^{4 \beta}\right) d_{R}{ }^{\beta}\right] W_{\mu}^{+}+\text {h.c. },
\end{aligned}
$$

where we have used Eqs. (4) and (5). The vector-like doublet quark induces FCNC in the up and down right-handed quark sectors, which for convenience we describe in the following way,

$$
\begin{aligned}
& z_{d}^{\alpha \beta} \equiv \sum_{i=1}^{3} D_{R}^{\alpha i} D_{R}^{\dagger i \beta}=\delta^{\alpha \beta}-D_{R}^{\alpha 4} D_{R}^{\dagger}{ }^{4 \beta}, \\
& z_{u}^{\alpha \beta} \equiv \sum_{i=1}^{3} U_{R}^{\alpha i} U_{R}^{\dagger i \beta}=\delta^{\alpha \beta}-U_{R}^{\alpha 4} U_{R}^{\dagger 4 \beta},
\end{aligned}
$$

by using unitarity of $U_{L / R}$ and $D_{L / R}$ matrices. $z_{q}(q=u, d)$ indicates the size of the induced FCNC's [9]. In the case where $\alpha=\beta$, one finds $z_{d}^{\alpha \alpha}=1-\left|D_{R}{ }^{\alpha 4}\right|^{2}$ and $z_{u}{ }^{\alpha \alpha}=1-\left|U_{R}{ }^{\alpha 4}\right|^{2}$. On the other hand, in the CC sector the right CKM matrix 
includes new effects. $U_{L}^{\alpha \delta} V_{L}^{\dagger \delta \beta}$ and $U_{R}^{\alpha 4} V_{R}^{\dagger 4 \beta}$ show the left and right CKM matrices in Eq. (10).

$$
\begin{aligned}
V_{L C K M}{ }^{\alpha \beta} & \equiv \sum_{\rho=1}^{4} U_{L}^{\alpha \rho} D_{L}^{\dagger \rho \beta}, \\
V_{R C K M}{ }^{\alpha \beta} & \equiv U_{R}^{\alpha 4} D_{R}^{\dagger}{ }^{4 \beta} .
\end{aligned}
$$

We use the ratio of the elements of left and right CKM matrix for convenience to make comparison with the SM's one and give the bounds from the experimental results. The ratio must be defined as

$$
\begin{aligned}
V^{\alpha \beta} & \equiv \frac{V_{R C K M^{\alpha \beta}}}{V_{L C K M^{\alpha \beta}}} \\
& =\left|\frac{V_{R C K M^{\alpha \beta}}}{V_{L C K M}{ }^{\alpha \beta}}\right| e^{i \theta_{\alpha \beta}},
\end{aligned}
$$

where

$$
\theta_{\alpha \beta} \equiv \arg \left(\frac{V_{R C K M^{\alpha \beta}}}{V_{L C K M^{\alpha \beta}}}\right) .
$$

This phase factors should induce new $C P$ violation sources in the model as generally a $4 \times 4$ matrix has three phases. $\left|V^{\alpha \beta}\right|$ indicates the size of the violation of the $V-A$ structure in the theory. Note that in general $V_{R C K M}$ is not a unitary matrix, while $V_{L C K M}$ retains its unitarity. This point is differs from the singlet vector-like quark model [9, 11] which violates the unitarity of $V_{L C K M}$.

Substituting the above relations into Eq. (11), the Lagrangian for the neutral and charged Higgs sector becomes,

$$
\begin{aligned}
& \mathcal{L}_{H, \chi^{0}}=-\frac{g}{2 M_{W}} \bar{q}^{\alpha} z_{q}{ }^{\alpha \beta} m_{q}^{\beta} R q^{\beta}\left(H \mp i \chi^{0}\right)+\text { h.c. }, \\
& \mathcal{L}_{\chi^{ \pm}}=\frac{g}{\sqrt{2} M_{W}} \bar{u}^{\alpha} V_{L C K M}{ }^{\alpha \beta} {\left[\left(1-V^{\alpha \beta} \frac{m_{d}^{\beta}}{m_{u}^{\alpha}}\right) m_{u}^{\alpha} L\right.} \\
&\left.-\left(1-V^{\alpha \beta} \frac{m_{u}^{\alpha}}{m_{d}^{\beta}}\right) m_{d}^{\beta} R\right] d^{\beta} \chi^{+}+\text {h.c. }
\end{aligned}
$$

where the upper sign is for $q=u$ and the lower one is for $q=d$. The same procedure gives the Lagrangian for the gauge boson quark sector,

$$
\mathcal{L}_{A}=g \sin \theta_{W} Q_{q} \bar{q}^{\alpha} \gamma_{\mu} q^{\alpha} A^{\mu}
$$




$$
\begin{aligned}
\mathcal{L}_{Z} & = \pm \frac{g}{2 \cos \theta_{W}} \bar{q}^{\alpha} \gamma_{\mu}\left[g_{q} \delta^{\alpha \beta} L \pm\left(g_{q} \delta^{\alpha \beta}-z_{q}^{\alpha \beta}\right) R\right] q^{\beta} Z^{\mu}, \\
\mathcal{L}_{W^{ \pm}} & =\frac{g}{\sqrt{2}} \bar{u}^{\alpha} V_{L C K M}{ }^{\alpha \beta} \gamma_{\mu}\left(L+V^{\alpha \beta} R\right) d^{\beta} W^{\mu+}+\text { h.c. . }
\end{aligned}
$$

Here $\theta_{W}$ is the Weinberg angle, $g_{q} \equiv\left(1 \mp 2 Q_{q} \sin ^{2} \theta_{W}\right)$, and $Q_{q}$ is the electric charge $\left(Q_{u}=2 / 3\right.$ and $\left.Q_{d}=-1 / 3\right)$. Therefore, in the present model, the violation of the $V-A$ structure appears in the CC sector (Eqs. (18) and (21)), while the FCNC's appear in the neutral-current(NC) sector (Eqs. (17) and (20)). It is clear that when one puts $V^{\alpha \beta}=0$ and $z_{q}^{\alpha \beta}=\delta^{\alpha \beta}$, the SM Lagrangian is restored.

In this model there are many newly introduced parameters. Hence, for simplicity, we make the following assumptions: $(\mathrm{A})$ the SM-like $3 \times 3$ matrix is retained for the left-handed CKM matrix, i.e.,

$$
V_{L C K M} \sim\left(\begin{array}{cc}
V_{C K M} & -\times \\
\times & 1
\end{array}\right)
$$

and (B) there are right-handed mixings among the heavy quarks but not among the light quarks. This can be realized by using a special form of the unitary matrices $U_{R}$ and $D_{R}$ as follows:

$$
\sim\left(\begin{array}{cccc}
1 & \times & \times & \times \\
\times & 1 & \times & \times \\
\times & \times & \cos \theta_{U / D} & -\sin \theta_{U / D} \\
\times & \times & \sin \theta_{U / D} & \cos \theta_{U / D}
\end{array}\right),
$$

which permits possible large mixings between the third and fourth generations of right-handed quarks. ' $\times$ ' denotes a small $(\ll 1)$ but non-zero element.

\section{CONSTRAINT FROM $R_{b}$}

Ref. [7] showed that there are regions allowed by the measurement of $R_{b}$ for nonzero values of the mixing parameters between the third and the fourth generations. The value of the parameter $\left|D_{R}^{34}\right|^{2}$ had to be larger than $4 \sin ^{2} \theta_{W} / 3$.

$R_{b}$ is defined as $R_{b} \equiv \Gamma(Z \rightarrow b \bar{b}) / \Gamma(Z \rightarrow$ hadrons $)$. An estimate for the upperbound of $\left|D_{R}^{34}\right|$ can be extracted [6, 0], that is

$$
R_{b}=\frac{\Gamma_{b b}}{\Gamma_{u u}+\Gamma_{c c}+\Gamma_{d d}+\Gamma_{s s}+\Gamma_{b b}},
$$




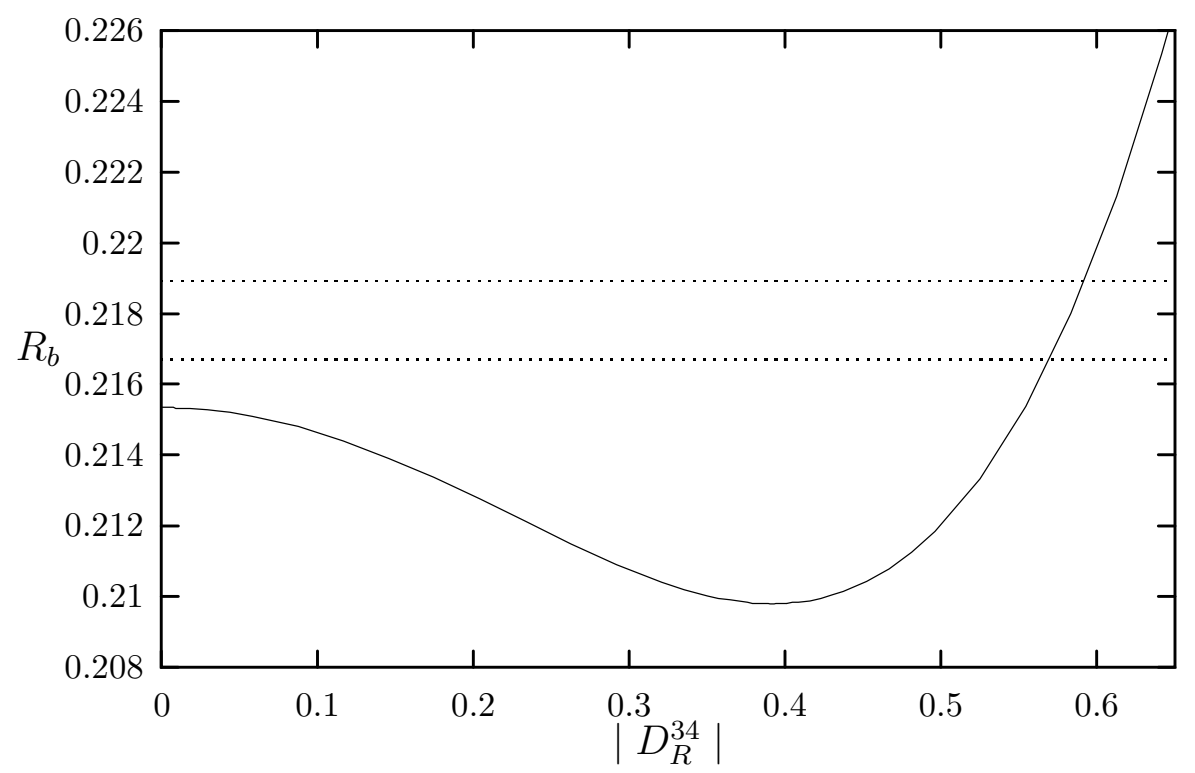

Figure 1: $R_{b}$ as a function of $\left|D_{R}^{34}\right|$

where

$$
\Gamma_{q^{i} q^{i}} \equiv\left(g_{q}^{i}+\delta_{S M} q^{i} q^{i}\right)^{2}+\left(g_{q}^{i}-z_{q}^{q^{i} q^{i}}\right)^{2}
$$

with $\delta_{S M} q^{i} q^{i}$ is the one-loop corrections of $Z \rightarrow q^{i} \bar{q}^{i}$ within the SM, and all of the fermion masses are neglected. $\left|D_{R}^{34}\right|$ appears in eqs.(25) through $z_{d}^{b b}$ according to Eqs. (11). Since $\delta_{S M} q^{i} q^{i}$, s are insignificant except for $q^{i}=b$, in the numerical calculations we will keep the one-loop correction only in $\Gamma_{b b}$ [3]. From Eq. (25),

$$
\Gamma_{b b}=\left(1-\frac{2}{3} \sin ^{2} \theta_{W}+\delta_{S M}^{b b}\right)^{2}+\left(-\frac{2}{3} \sin ^{2} \theta_{W}+\left|D_{R}^{34}\right|^{2}\right)^{2} .
$$

In Fig. 11 $R_{b}$ is shown as a function of $\left|D_{R}{ }^{34}\right|$ for $R_{c}=0.172$ and $\delta_{S M}^{b b}=-0.011$. From the figure, we find the parameter $\left|D_{R}^{34}\right|$ must be larger than 0.55 when the measurement values of $R_{b}$ is larger than the SM prediction. For $R_{b}=0.2178 \pm 0.0011$ and $R_{c}=0.172$ [5] the bound is

$$
0.57<\left|D_{R}^{34}\right|<0.59
$$

Even if the experiment of $R_{b}$ is consistent with the SM, the figure shows that the region for large value of $\left|D_{R}^{34}\right|$ may be survive. 
However, if $\left|D_{R}^{34}\right|$ has a large value, from Eq. (14), $V_{R C K M}^{33}$ may also be large, or $U_{R}^{34}$ may be very small.

\section{RADIATIVE $B$ DECAYS}

Next, We consider radiative $B$ decays in the model. After calculating the relevant Feynman diagrams including the neutral Higgs contributions, we find the amplitude for on-shell $B \rightarrow X_{d_{l}} \gamma$ up to second order in the external momenta as below,

$$
\begin{aligned}
T=\frac{G_{F} e}{8 \sqrt{2} \pi^{2}} V_{L C K M}^{*}{ }^{t d_{l}} V_{L C K M}{ }^{t b} Q_{u} \bar{d}_{l}\left(p^{\prime}\right) \\
\quad \times\left[\gamma_{\mu}, \gamma_{\nu} q^{\nu}\right]\left(F_{L}\left(m_{d_{l}}\right) m_{d_{l}} L+F_{R}\left(m_{d_{l}}\right) m_{b} R\right) b(p) \epsilon^{\mu}
\end{aligned}
$$

with

$$
\begin{aligned}
& F_{L}\left(m_{d_{l}}\right)=\sum_{i=t, u^{4}} \frac{V_{L C K M}^{*}{ }^{i d_{l}} V_{L C K M^{i b}}}{V_{L C K M}^{*}{ }^{t d_{l}} V_{L C K M}{ }^{t b}}\left[\left(1+V^{* i d_{l}} V^{i b} \frac{m_{b}}{m_{d_{l}}}\right) F_{1}\left(x_{i}\right)\right. \\
& \left.+V^{* i d_{l}} F_{2}\left(x_{i}, m_{d_{l}}\right)+\left(1-V^{* i d_{l}} \frac{m_{i}}{m_{d_{l}}}\right) F_{3}\left(x_{i}\right)\right] \\
& -\frac{Q_{d}}{Q_{u}} \frac{z_{d}^{d_{l} b}}{V_{L C K M}^{*}{ }^{t d_{l}} V_{L C K M}{ }^{t b}}\left[g_{d} F_{4}\left(r_{d_{l}}\right)+\left|D_{R}^{44}\right|^{2}\left(F_{5}\left(r_{d^{4}}, w_{d^{4}}\right)+F_{6}\left(r_{d^{4}}\right)\right)\right. \\
& \left.+\frac{1}{3}\left(\left|D_{R}{ }^{14}\right|^{2}+\left|D_{R}^{24}\right|^{2}+\left|D_{R}^{34}\right|^{2}\right)\right], \\
& F_{R}\left(m_{d_{l}}\right)=\sum_{i=t, u^{4}} \frac{V_{L C K M}^{*}{ }^{i d_{l}} V_{L C K M}{ }^{i b}}{V_{L C K M}^{*}{ }^{t d_{l}} V_{L C K M}{ }^{t b}}\left[\left(1+V^{* i d_{l}} V^{i b} \frac{m_{d_{l}}}{m_{b}}\right) F_{1}\left(x_{i}\right)\right. \\
& \left.+V^{i b} F_{2}\left(x_{i}, m_{b}\right)+\left(1-V^{i b} \frac{m_{i}}{m_{b}}\right) F_{3}\left(x_{i}\right)\right] \\
& -\frac{Q_{d}}{Q_{u}} \frac{z_{d}^{d_{l} b}}{V_{L C K M}^{*}{ }^{t d_{l}} V_{L C K M}{ }^{t b}}\left[g_{d} F_{4}\left(r_{b}\right)+\left|D_{R}^{44}\right|^{2}\left(F_{5}\left(r_{d^{4}}, w_{d^{4}}\right)+F_{6}\left(r_{d^{4}}\right)\right)\right. \\
& \left.+\frac{1}{3}\left(\left|D_{R}{ }^{14}\right|^{2}+\left|D_{R}^{24}\right|^{2}+\left|D_{R}^{34}\right|^{2}\right)\right],
\end{aligned}
$$

where $x_{i} \equiv\left(m_{i} / M_{W}\right)^{2}, r_{\alpha} \equiv\left(m_{\alpha} / M_{Z}\right)^{2}$ and $w_{\alpha} \equiv\left(m_{\alpha} / M_{H}\right)^{2}$. For simplicity we have employed the relations

$$
z_{d}^{\dagger d_{l} \alpha} z_{d}^{\alpha b} \simeq \begin{cases}0 & \text { for } \alpha=d_{l}, b \\ -z_{d} d_{l} b\left|D_{R}{ }^{\alpha 4}\right|^{2} & \text { for } \alpha \neq d_{l}, b\end{cases}
$$

by using Eq. (11) and the approximation that $r_{d_{i}} \simeq w_{d_{i}} \simeq 0$. Then $F_{5}\left(r_{d^{i}}, w_{d^{i}}\right) \sim 0$ and $F_{6}\left(r_{d^{i}}\right) \sim 1 / 3$. On the other hand, $F_{5}\left(r_{d^{4}}, w_{d^{4}}\right)$ and $F_{6}\left(r_{d^{4}}\right)$ could be significant. 
One assumption here is that $V_{R C K M} M^{u^{i} d^{j}}$ for $u^{i}=u, c$ and $d^{j}=d_{l}, b$ are negligible compared with the other elements. This assumption and how it is realized will be discussed in detail in the next section. On the other hand, since the unitarity of $V_{L C K M}$ is still retained, the relation

$V_{L C K M}^{*}{ }^{u d_{l}} V_{L C K M}{ }^{u b}+V_{L C K M}^{*}{ }^{c d_{l}} V_{L C K M}{ }^{c b}=-V_{L C K M}^{*}{ }^{t d_{l}} V_{L C K M}{ }^{t b}-V_{L C K M}^{*}{ }^{u^{4} d_{l}} V_{L C K M}{ }^{u^{4} b}$

is valid. Similar to Eq. (15), there are also phase factors which induce $C P$ violation from the mixings of FCNC, that is

$$
\frac{z_{d} d_{l} b}{V_{L C K M}^{*}{ }^{t d_{l}} V_{L C K M}{ }^{t b}}=\left|\frac{z_{d}^{d_{l} b}}{V_{L C K M}^{*}{ }^{t d_{l}} V_{L C K M}{ }^{t b}}\right| e^{i \theta_{d_{l}}},
$$

with

$$
\theta_{d_{l}} \equiv \arg \left(\frac{z_{d}^{d_{l} b}}{V_{L C K M}^{*}{ }^{t d_{l}} V_{L C K M}{ }^{t b}}\right) .
$$

Each function in Eqs. (29) and (30) includes the contributions of each diagram. That is, $F_{1,3}, F_{2}, F_{4,6}$ and $F_{5}$ come from the $W^{ \pm}, \chi^{ \pm}, Z$ and neutral Higgs $\left(H, \chi^{0}\right)$ exchange diagrams respectively. These functions are given by

$$
\begin{aligned}
F_{1}(x) & =x\left[\frac{-113+151 x+28 x^{2}}{72(1-x)^{3}}-\frac{8+2 x-21 x^{2}}{12(1-x)^{4}} \ln x\right], \\
F_{2}\left(x, m, m_{i}\right)= & 4 \frac{1-x^{2}+2 x \ln x}{3(1-x)^{3}}-2 \frac{m_{i}}{m} \frac{3-4 x+x^{2}+2 \ln x}{(1-x)^{3}}, \\
F_{3}(x)= & x\left[\frac{25-53 x+22 x^{2}}{36(1-x)^{3}}+\frac{4-8 x+3 x^{2}}{6(1-x)^{4}} \ln x\right], \\
F_{4}(x)= & \frac{-10+5 x-11 x^{2}}{6(1-x)^{3}}-\frac{3-4 x}{(1-x)^{4}} x \ln x \\
F_{5}(x, y)= & x\left[\frac{-20+19 x-5 x^{2}}{24(1-x)^{3}}-\frac{2-x}{4(1-x)^{4}} \ln x\right] \\
& -y\left[\frac{-16+29 y-7 y^{2}}{24(1-y)^{3}}-\frac{2-3 y}{4(1-y)^{4}} \ln y\right] \\
F_{6}(x)= & \frac{4-9 x-5 x^{3}-6 x(1-2 x) \ln x}{12(1-x)^{4}} .
\end{aligned}
$$

From these results, one can predict the possible enhancements for the violations of $V-A$ structure as well as FCNC. The enhancement of the violations of $V-A$ structure is possibly large as there are some terms proportional to the third- and 
fourth-generation up-quark masses which are assumed to be large $\left(m_{u^{4}}>m_{t} \sim\right.$ $174(\mathrm{GeV}))$. Also in the present model one can not neglect the left-handed term of the amplitude as is always done in the simplified SM calculations with same reason.

\section{CONSTRAINTS FOR THE MIXINGS AND MASSES}

Before doing further evaluation, we impose experimental constraints on the mixing parameters and vector-like quark masses of Eqs. (29) and (30). First, consider the mixing parameter $z_{d} d_{l} b$ which indicates the FCNC in the $d_{l} b$ sector. Using the experimental results [13, 14], one finds the upper-bounds for them as,

$$
\left|z_{d}{ }^{d_{l} b}\right| \leq 1.7 \times 10^{-3}
$$

from $\operatorname{Br}\left(B \rightarrow X_{d_{l}} \mu^{+} \mu^{-}\right) \leq 5 \times 10^{-5}$ under an assumption that the Z-mediated tree-level Feynman diagram is dominant [11]. For $i=t$ and $d_{l}=s$, one finds the ratio of mixing in Eqs. (29) and (30) as follows:

$$
\left|\frac{z_{d}^{s b}}{V_{L C K M}^{*}{ }^{t s} V_{L C K M}{ }^{t b}}\right| \leq 4.0 \times 10^{-2},
$$

from the assumption (A) of Sec.⿴囗十 for left-handed CKM matrix elements, $\left|V_{L C K M}{ }^{t s}\right| \sim$ $\left|V_{L C K M}{ }^{c b}\right| \sim 0.042$ and $\left|V_{L C K M}{ }^{t b}\right| \sim 1$ [13]. We find that the contribution from the $z_{d}^{d^{l} b}\left(d^{l} \neq b\right)$ term is small enough to neglect.

Our next task is determining the bound on the vector-like quark masses, $m_{u^{4}}$ and $m_{d^{4}}$, and the mixings $\left|V^{t d^{i}}\right|$ and $\left|V^{u^{4} d^{i}}\right|$ which can be done by considering the experiment measurement of $B \rightarrow X_{s} \gamma$ process and the $T_{\text {new }}$ parameter. The ratio of branching ratio is given as,

$\frac{B r\left(b \rightarrow d_{l} \gamma\right)}{B r(b \rightarrow c l \nu)}=\frac{3 \alpha Q_{u}{ }^{2}}{2 \pi f\left(m_{c}{ }^{2} / m_{b}{ }^{2}\right)}\left|\frac{V_{L C K M}^{*}{ }^{t d_{l}} V_{L C K M}{ }^{t b}}{V_{L C K M}{ }^{c b}}\right|^{2}\left(\frac{m_{d_{l}}{ }^{2}}{m_{b}{ }^{2}} F_{L}\left(m_{d_{l}}\right)^{2}+F_{R}\left(m_{d_{l}}\right)^{2}\right)$.

which is normalized by the semileptonic decay $b \rightarrow c l \nu$ to reduce the uncertainties due to $m_{b}$ and the left CKM matrix element, especially for $d_{l}=s$. The phase space factor is

$$
f(y) \equiv 1-8 y+8 y^{3}-y^{4}-12 y^{2} \ln y,
$$




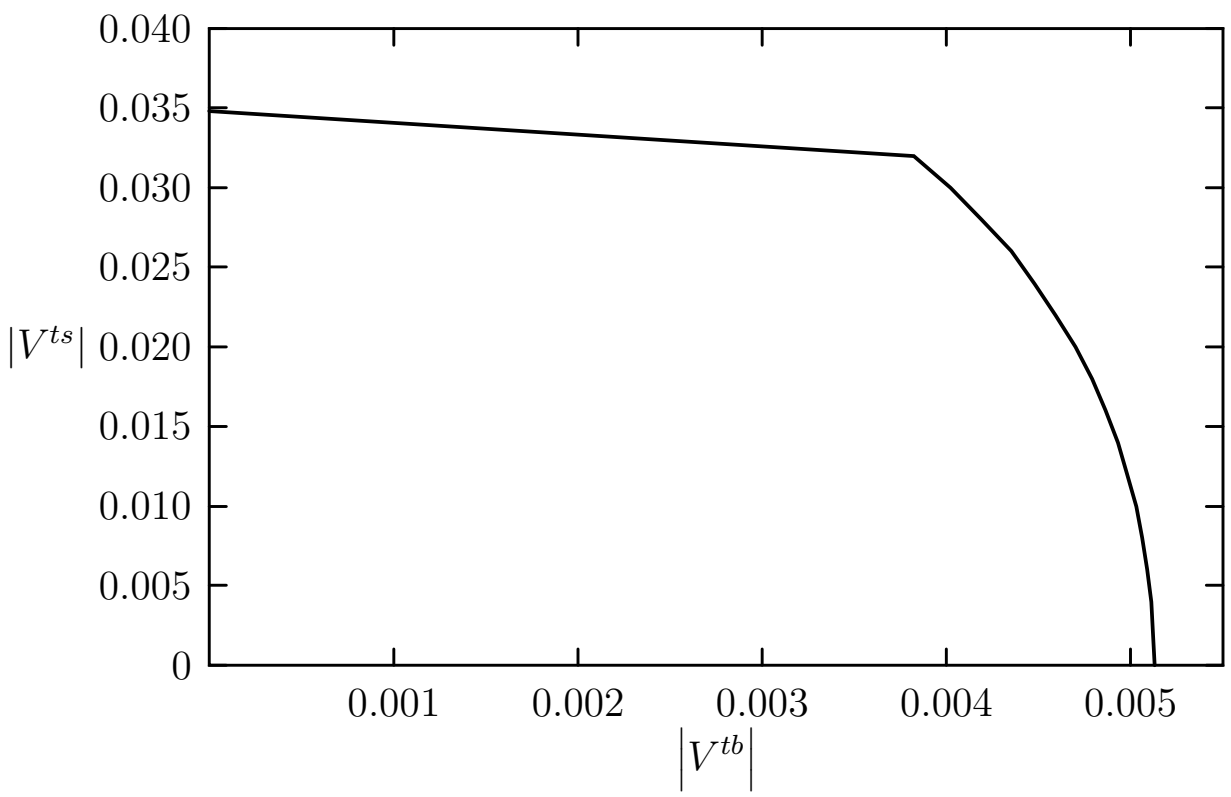

Figure 2: The allowed upper-bounds for $\left|V^{t s}\right|$ and $\left|V^{t b}\right|$ from $b \rightarrow s \gamma$.

and equals 0.520 for $m_{c} / m_{b}=0.3$ [15]. Note that the left handed term is kept because there is a possible enhancement due to the heavy quark mass from the $m_{i} / m$ term in Eq. (36).

Now we can determine the upper-bounds for $\left|V^{t s}\right|$ and $\left|V^{t b}\right|$. Fortunately, the $b \rightarrow s \gamma$ process, the contribution of FCNC with the upper-bound in Eq. (42) is negligible 11]. By putting $V_{L C K M} u^{4} s=0$ at once and using the value, $\operatorname{Br}(B \rightarrow$ $\left.X_{s} \gamma\right)=5 \times 10^{-4}, \operatorname{Br}\left(B \rightarrow X_{c} l \nu\right)=0.12$ and the maximum phase factors $\theta_{s}=$ $\theta_{t s}=0$, we find the upper-bounds as depicted in Fig. 2. Here we find that the $\left|V^{t b}\right| \sim\left|V_{R}^{t b}\right|$ must be less than 0.0052. These results are reasonable because the $b \rightarrow s \gamma$ process can be explained within the SM, hence the contribution of the new physics should be small.

We find that the upper bound of $\left|V_{R}^{t b}\right|$ from $b \rightarrow s \gamma$. Hence, we can find the upper bound of $\left|U_{R}^{34}\right|$ by using the relation for the right CKM matrix (14) and these bounds from $b \rightarrow s \gamma$ and $R_{b}(27)$.

$$
\left|U_{R}^{34}\right|<0.0092
$$

Lastly, we are going to use $T_{\text {new }}$ to determine the allowed regions of $\left|U_{R}^{34}\right|$. The 


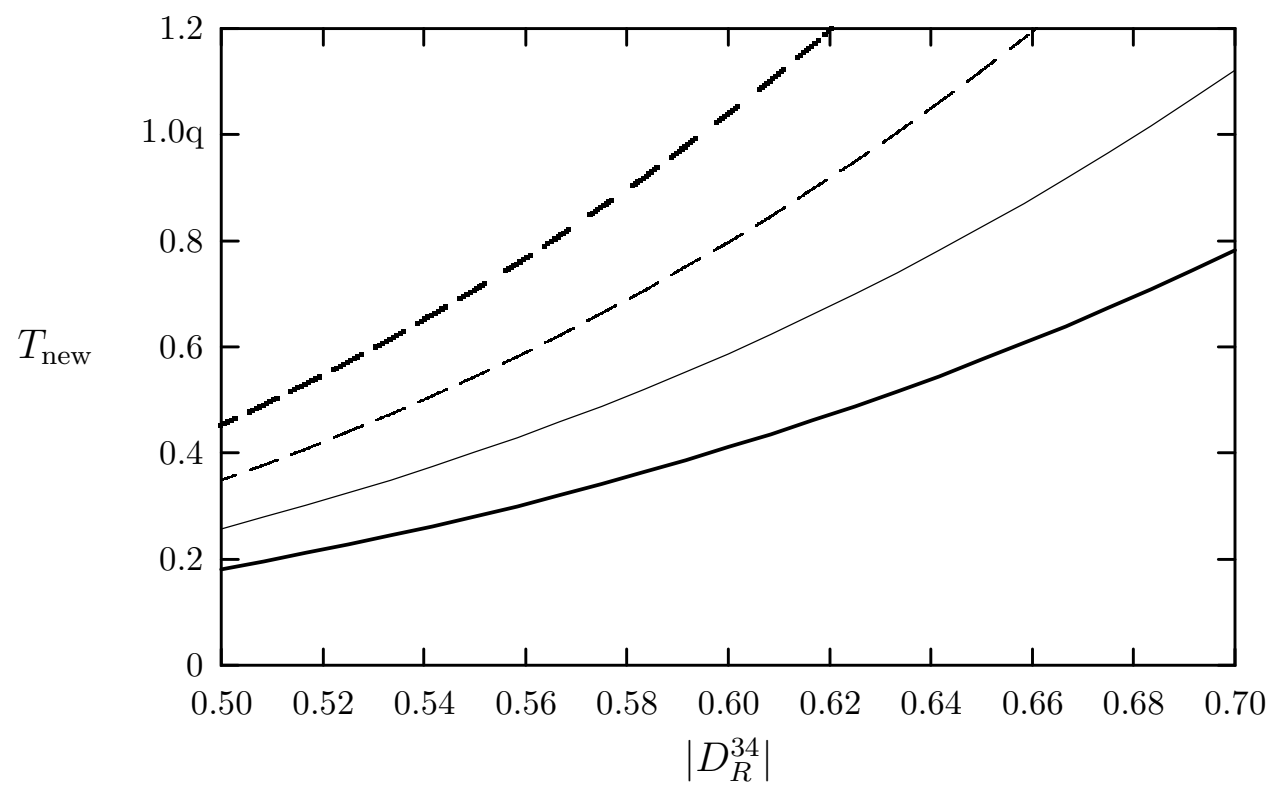

Figure 3: The dependence of $T_{\text {new }}$ on $\left|D_{R}^{34}\right|$ for $m_{d^{4}}=250(\mathrm{GeV})$ (thick solid line), $m_{d^{4}}=300(\mathrm{GeV})$ (thin solid line), $m_{d^{4}}=350 \mathrm{GeV}$ ) (thin dashed line) and $m_{d^{4}}=400$ $\mathrm{GeV})$ (thick dashed line) with $\left|V_{L C K M}{ }^{t d^{4}}\right|=0.01$.

interactions between the gauge bosons and the quarks which contribute to the $T$ parameter are,

$$
\begin{aligned}
\mathcal{L}_{W^{1,3}}= & \frac{g}{2}\left\{ \pm \bar{q}^{\alpha} \gamma^{\mu} W_{\mu}^{3}\left[\delta^{\alpha \beta} L+\left(\delta^{\alpha \beta}-z_{q}^{\alpha \beta}\right) R\right] q^{\beta}\right. \\
& \left.-\bar{u}^{\alpha} \gamma^{\mu} W_{\mu}^{1} V_{L C K M}{ }^{\alpha \beta}\left(L+V^{\alpha \beta} R\right) d^{\beta}+\text { h.c. }\right\} .
\end{aligned}
$$

The $T$ parameter becomes

$$
\begin{aligned}
T= & \frac{N_{c}}{32 \pi M_{Z}^{2} \sin ^{2} \theta_{W} \cos ^{2} \theta_{W}} \sum_{\alpha, \beta}^{4}\left\{2\left|V_{L C K M}{ }^{\alpha \beta}\right|^{2} f\left(m_{u^{\alpha}}, m_{d^{\beta}}\right)\left(1+\left|V^{\alpha \beta}\right|^{2}\right)\right. \\
& -16 g\left(m_{u^{\alpha}}, m_{d^{\beta}}\right) V_{L C K M}{ }^{\alpha \beta} \operatorname{Re}^{\alpha \beta} \\
& -f\left(m_{u^{\alpha}}, m_{u^{\beta}}\right)\left[\delta^{\alpha \beta}+\left(\delta^{\alpha \beta}-z_{u}{ }^{\alpha \beta}\right)^{2}\right]-f\left(m_{d^{\alpha}}, m_{d^{\beta}}\right)\left[\delta^{\alpha \beta}+\left(\delta^{\alpha \beta}-z_{d}^{\alpha \beta}\right)^{2}\right] \\
& \left.-8 h\left(m_{u^{\alpha}}\right)\left|U_{R}{ }^{\alpha 4}\right|^{2}-8 h\left(m_{d^{\alpha}}\right)\left|D_{R}{ }^{\alpha 4}\right|^{2}\right\},
\end{aligned}
$$

from the definitions of $T$ parameter presented by Ref. [16]. $N_{c}$ is the number of color. These auxiliary functions are defined as,

$$
f(x, y) \equiv x^{2}+y^{2}-2 \frac{x^{4} \ln x^{2}-y^{4} \ln y^{2}}{x^{2}-y^{2}},
$$




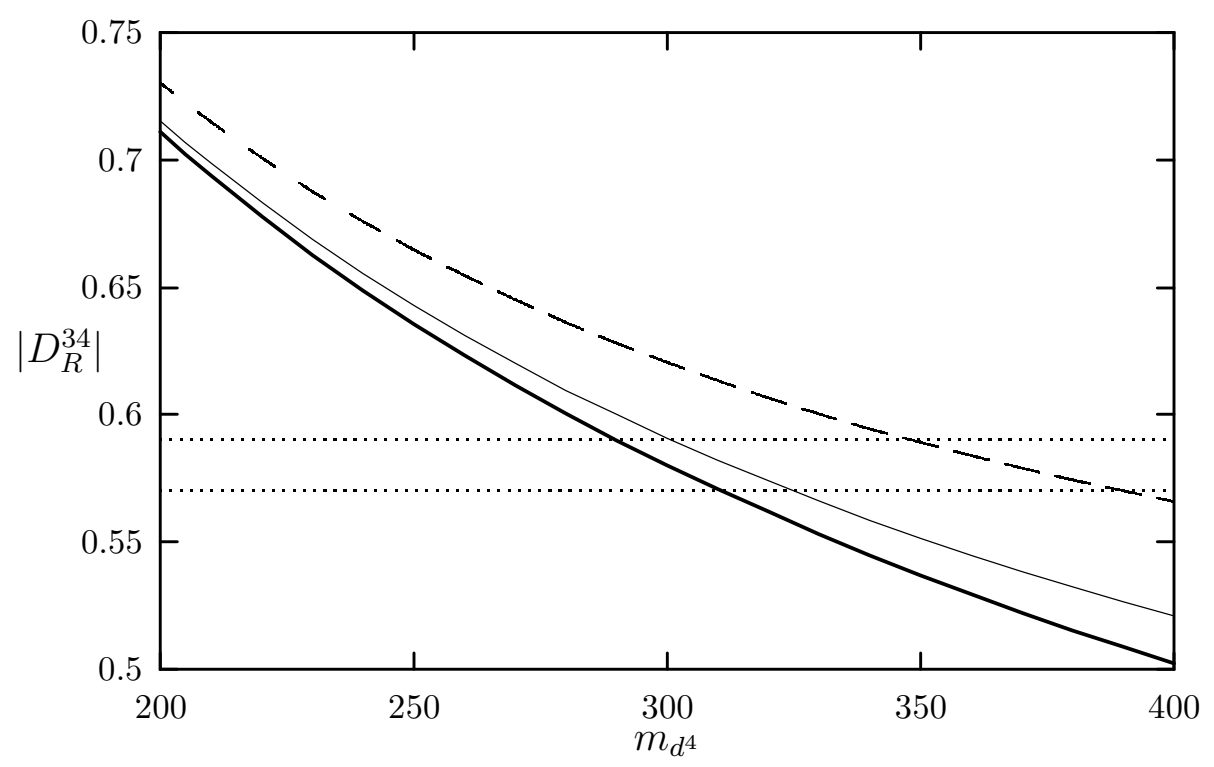

Figure 4: The upper bound of $\left|D_{R}^{34}\right|$ and $m_{d^{4}}$ from the bound of $T_{N E W}$ for $\left|V_{L C K M}{ }^{t d^{4}}\right|=0$ (thick line), $\left|V_{L C K M^{t d^{4}}}\right|=0.01$ (thin line), $\left|V_{L C K M}{ }^{t d^{4}}\right|=0.02$ (dashed line). The dotted lines show the bound,eq.(27) from $R_{b}$.

$$
\begin{aligned}
g(x, y) & \equiv x y\left(1-\frac{x^{2} \ln x^{2}-y^{2} \ln y^{2}}{x^{2}-y^{2}}\right) \\
h(x) & \equiv x^{2} \ln x^{2} .
\end{aligned}
$$

The $T_{\text {new }}$ parameter is found by subtracting the SM contribution. According to Eqs. (6), (13) and (14), one finds the following mass relations,

$$
\begin{aligned}
\delta^{\alpha \beta} m_{d}{ }^{\beta} D_{R}{ }^{\beta 4} D_{R}^{\dagger}{ }^{4 \sigma} & =V_{L C K M}^{\dagger}{ }^{\alpha \beta} m_{u}{ }^{\beta} V_{R C K M}{ }^{\beta \sigma}, \\
\delta^{\alpha \beta} m_{u}{ }^{\beta} U_{R}{ }^{\beta 4} U_{R}^{\dagger} & =V_{L C K M}{ }^{\alpha \beta} m_{d}{ }^{\beta} V_{R C K M}^{\dagger}{ }^{\beta \sigma} .
\end{aligned}
$$

Both equations yield a relation between $m_{u^{4}}$ and $m_{d^{4}}$, and $U_{R}^{34}\left(\sim \sin \theta_{U}\right)$ and $D_{R}^{34}(\sim$ $\left.\sin \theta_{D}\right)$ under the assumptions (A) and (B) ( Eq.(22) and Eq.(23) ).

$$
\begin{aligned}
m_{u^{4}} & =\frac{1}{\cos \theta_{U}}\left(-m_{b} V_{L C K M}{ }^{t d^{4}} \sin \theta_{D}+m_{d^{4}} \cos \theta_{D}\right), \\
\sin \theta_{U} & =\frac{m_{b}}{m_{t}} \sin \theta_{D}-\frac{m_{d^{4}}}{m_{t}} V_{L C K M}{ }^{t d^{4}} \cos \theta_{D}
\end{aligned}
$$

Using these relations, we can simplify our expression for $T_{\text {new }}$ and plot the figures. Fig.3 shows $T_{\text {new }}$ vs $\left|D_{R}^{34}\right|$. Fig.4 shows the upper bound on $\left|D_{R}^{34}\right|$ and $m_{d^{4}}$ from the 


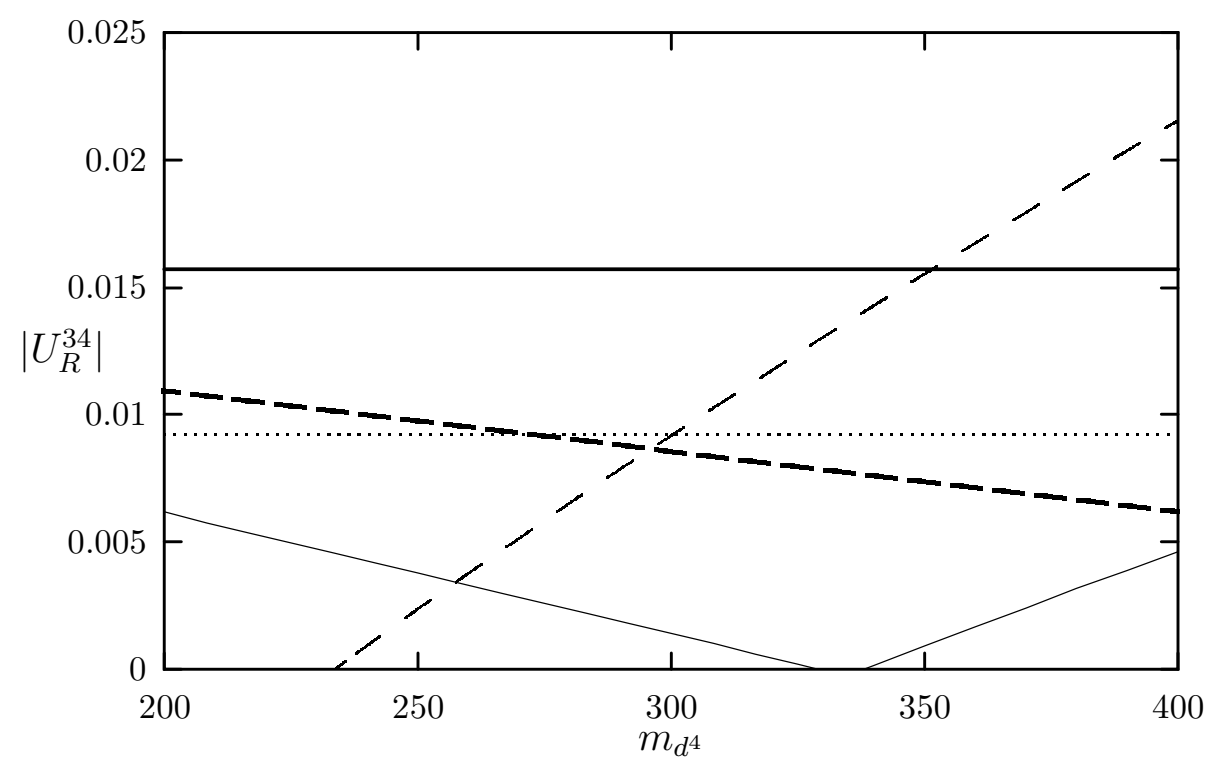

Figure 5: The lower bound of $\left|U_{R}^{34}\right|$ from the bound $\left|D_{R}^{34}\right|>0.55$ and the upper bound of $T_{N E W}$ for $\left|V_{L C K M}{ }^{t d^{4}}\right|=0$ (thick line), $\left|V_{L C K M}{ }^{t d^{4}}\right|=0.005$ (thick dashed line), $\left|V_{L C K M}{ }^{t d^{4}}\right|=0.01$ (thin line), $\left|V_{L C K M}{ }^{t d^{4}}\right|=0.02$ (thin dashed line). The dotted line show the upper bound from $R_{b}$ and $b \rightarrow s \gamma($ Eq. (45)).

upper bound of $T_{\text {new }},\left(T_{\text {new }}<0.55\right.$ for $m_{t}=175 \mathrm{GeV}$ and $\left.m_{H}=1 \mathrm{TeV}\right)$ 13, 17. By using the eq. (54), we obtain the lower bound on $\left|U_{R}^{34}\right|$ from the lower bound of $\left|D_{R}^{34}\right|$ (> 0.55) that is the bound when there is the deviation from the SM prediction in $R_{b}$.(See the Fig.1.) In the figures we put $m_{t}=175(\mathrm{GeV}), m_{b}=5(\mathrm{GeV})$ and $\left|V_{L C K M}{ }^{t d^{4}}\right| \sim\left|V_{L C K M} u^{u^{4} b}\right|$, and also neglect tiny contributions from the mixings between light-quarks and the fourth generation quarks.

Unfortunately, after calculating $T_{\text {new }}$ we find that there is no allowed region for $T_{\text {new }}<0.55$ for any values of vector-like quark masses $\left(m_{u^{4}}\right.$ and $\left.m_{d^{4}}\right)$ consistent with assumption (A) of Sec. [1] of left-handed CKM matrix. When $\left|V_{L C K M}^{t d^{4}}\right|=0$, Fig. \# shows that $m_{d}^{4}$ is less than about $300 \mathrm{GeV}$, and Fig. 5 shows that there is no region to satisfy the bound of $\left|U_{R}^{34}\right|$, Eq. (45). Therefore, let us use more general of the LCKM matrix for further analysis. If $\left|V_{L C K M}{ }^{t d^{4}}\right| \neq 0$, in Figs. 田 and 5, the allowed regions for vector-like quark mass $m_{d^{4}}$ for some values of $\left|U_{R}^{34}\right|,\left|D_{R}^{34}\right|$ and $\left|V_{L C K M}{ }^{t d^{4}}\right|$ are plotted by using Eqs. (47), (53) and (54). 


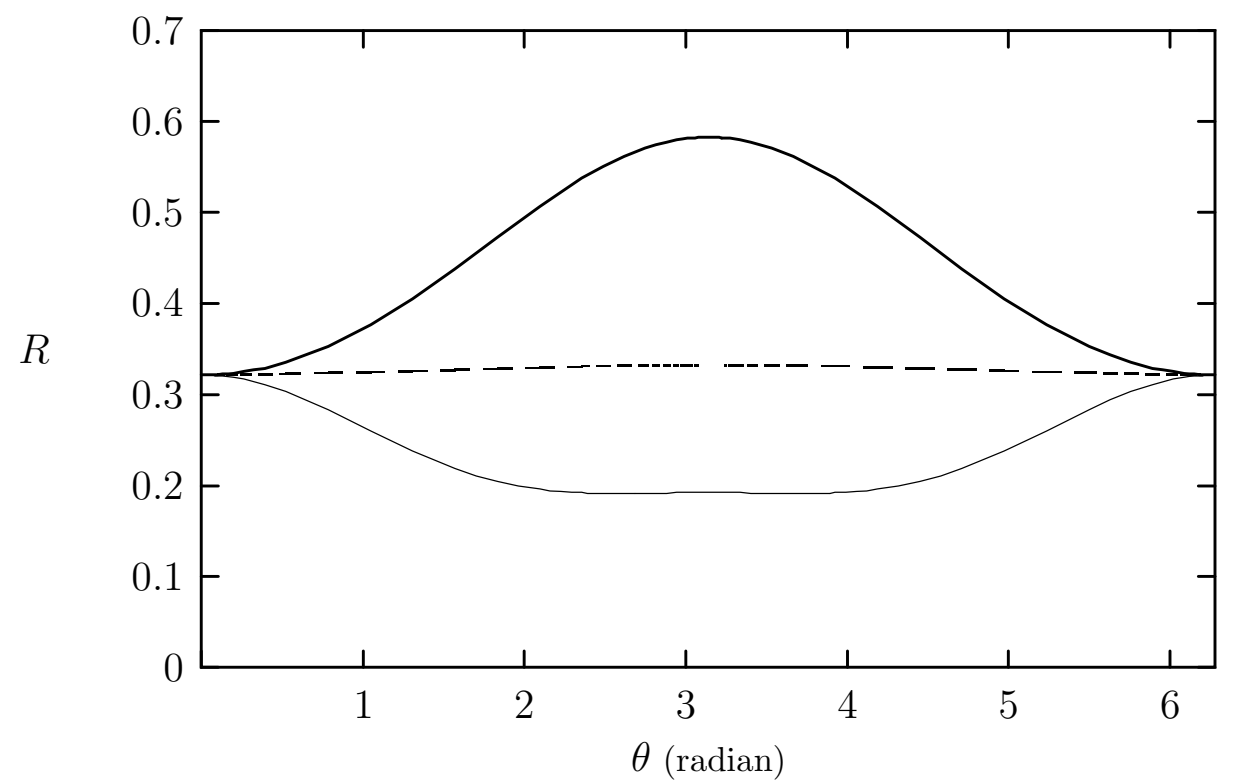

Figure 6: $R$ as a function of $\theta_{u^{4} b}$ (thick line) with $\theta_{d}=\theta_{u^{4} d}=0, \theta_{d}$ (dashed line) with $\theta_{u^{4} b}=\theta_{u^{4} d}=0$ and $\theta_{u^{4} d}$ (thin line) with $\theta_{u^{4} b}=\theta_{d}=0$.

\section{EFFECTS IN THE RATIO $R$}

Now we are ready to evaluate the contributions of the FCNC's and the violation of $V-A$ structure to the ratio $R$. Note that, in the present paper, the QCD corrections are not included. $R$ is defined as

$$
R \equiv \frac{\operatorname{Br}(b \rightarrow d \gamma)}{\operatorname{Br}(b \rightarrow s \gamma)}
$$

Then, from Eq. (43),

$$
R=\left|\frac{V_{L C K M}{ }^{t d}}{V_{L C K M}{ }^{t s}}\right|^{2} \frac{\left(m_{d}^{2} / m_{b}^{2}\right) F_{L}\left(m_{d}\right)^{2}+F_{R}\left(m_{d}\right)^{2}}{\left(m_{s}{ }^{2} / m_{b}{ }^{2}\right) F_{L}\left(m_{s}\right)^{2}+F_{R}\left(m_{s}\right)^{2}} .
$$

The type $B \rightarrow X_{d} \gamma$ decay has not been observed yet, but upper-bounds have been obtained from the exclusive decays $B \rightarrow\left(\rho^{-}, \rho^{0}, \omega\right) \gamma$ [18]. Then, within the SM,

$$
\left|\frac{V_{L C K M^{t d}}}{V_{L C K M^{t s}}}\right|<\frac{1}{1.8} \text {, }
$$

has been extracted, and theoretically the ratio becomes $R<0.31$. We have to include all of FCNC's effects because the contributions are not negligible [11]. However, under the assumption and experimental bounds in the preceding section, the 
terms $\left|D_{R}{ }^{i 4}\right|^{2}$ can be neglected safely, since they are small compared to the diagonal ones.

According to the results of the preceding section, for further numerical calculations, we put $\left|D_{R}^{34}\right|=0.58,\left|U_{R}^{34}\right|=0.004, m_{d^{4}}=290(\mathrm{GeV}), \sin ^{2} \theta_{W}=0.234$, $M_{H}=500(\mathrm{GeV})$ and $m_{t}=174(\mathrm{GeV}), m_{b}=5(\mathrm{GeV})$. With these values, one finds respectively $\left|D_{R}{ }^{44}\right| \sim 0.815,\left|V^{t b}\right|=0.002,\left|V^{u^{4} b}\right|=0.006,\left|V^{t s}\right| \sim 0.033$ and $\left|V^{u^{4} s}\right| \sim 3.7$. On the other hand, rough order estimations by considering unitarity of $V_{L C K M}$ and Eq. (23), gives $\left|V^{u^{4} d}\right| \sim 0.01,\left|V_{L C K M}^{*}{ }^{u^{4} d} V_{L C K M}{ }^{u^{4} b} / V_{L C K M}^{*}{ }^{t d}\right| \sim 0.01$, $\left|V^{t d}\right| \sim 0.01$ and $\left|z_{d}^{d b} / V_{L C K M}{ }^{t d}\right| \sim 0.1$. In Fig. 6, the ratio is depicted as a function of the phases, $\theta_{d}, \theta_{u^{4} d}$ and $\theta_{u^{4} b}$. On the other hand, since the contributions from the terms of $V^{t d}$ and $V^{t b}$ are comparably small, the deviations due to its phase should be negligible.

\section{CONCLUSIONS}

The effects of extending the SM by including a vector-like doublet of quarks on the FCNC and the violation of $V-A$ structure on the ratio $R$ has been studied. Including the constraints from experimental results, we found that there will be significant deviations in $R$ even for small mixings.

To satisfy the constraints from $R_{b}, b \rightarrow s \gamma$ and the $T$ parameter on the vector-like

doublet model, $m_{d^{4}}$ must be less than about $350(\mathrm{GeV})$ and $\left|V_{L C K M}^{t d^{4}}\right| \neq 0$. However an allowed region still exists even if $\left|D_{R C K M}^{34}\right|$ has a large value.

Lastly, because the experimental constraint of Eq. (57) is not strong, improved data and the eventual detection of the type $B \rightarrow X_{d} \gamma$ decays will provide an important test of the SM and will further constrain the present model.

\section{ACKNOWLEDGMENT}

We would like to thank Dr.R.Szalapski for reading the manuscript and some kindly comments. L.T.H. expresses his gratitude to the Japanese Government for financially supporting his work under Monbusho Fellowship. 


\section{References}

[1] M. S. Alam et.al. (CLEO Collaboration), Phys. Rev. Lett. 74, 2885 (1995).

[2] F. Abe et.al. (CDF Collaboration), Phys. Rev. Lett. 76, 4675 (1996).

[3] F. Cornet, W. Hollik and W. Mosle, Nucl. Phys. B428, 61 (1994).

[4] ALEPH Collaboration, The XXVIII International Conference on High Energy Physics, ICHEP Warsaw, Poland, 25-31 July 1996.

[5] LEP Electroweak Working Group and the SLD Heavy Flavor Group, CERNPPE-96-183 (1996).

[6] P. Bamert, C. P. Burgess, J. M. Cline, D. London and E. Nardi, Phys. Rev. D54, 4275 (1996);

E. Ma, Phys.Rev. D53 2276(1996);

G. Bhattacharyya, G. C. Branco and W. S. Hou, Phys. Rev. D54, 2114 (1996);

C. H. V. Chang, D. Chang and W. Y. Keung, Phys. Rev. D54, 7051 (1996).

[7] T. Yoshikawa, Prog. Theor. Phys.96, 269 (1996).

[8] K. Fujikawa and A. Yamada, Phys. Rev. D49, 5890 (1994).

[9] G. C. Branco and L. Lavoura, Nucl. Phys. B278, 738 (1986);

G. C. Branco, T. Morozumi, P. A. Parada and M. N. Rebelo, Phys. Rev. D48,1167 (1993);

V. Barger, M. S. Berger and R. J. N. Philips, Phys. Rev. D52, 1663 (1995).

[10] L. Lavoura and J. P. Silva, Phys. Rev. D47, 2046 (1993).

[11] L. T. Handoko and T. Morozumi, Mod. Phys. Lett. A10, 309 (1995); Proceeding of International Workshop on B Physics (World Scientific, 1995) 314, edited by A. I. Sanda and S. Suzuki. 
[12] Gautam Bhattacharyya, G. C. Branco and Debajyoti Choudhury, Phys. Lett. B366, 487 (1994).

[13] Particle Data Group, Phys. Rev. D54 (1996) 1.

[14] C. Aljabar et.al. (UA1 Collaboration), Phys. Lett. B262, 163 (1991) .

[15] R. Grigjanis, P. J. O'Donnell, Mark Suthe rland and Henri Navalet, Phys. Rep. 228, 93 (1993).

[16] B. Holdom and J. Terning, Phys. Lett. B247, 88 (1990);

M. Golden and L. Randall, Nucl. Phys. B361,3 (1991);

M. E. Peskin and T. Takeuchi, Phys. Rev. Lett. 65, 964 (1990); Phys. Rev. D46, 381 (1992).

[17] K. Hagiwara, Proceeding of YITP International Seminar '95 : From the Standard Model to Grand Unified Theories, Kyoto, Japan, 21-25 Aug 1995, Prog. Theor. Phys. Supp. 123 (1996) 173.

[18] R. A. Poling, 1994 International Workshop on B Physics, edited by A. I. Sanda and S. Suzuki, World Scientific (1995) 91. 